\title{
SYNTHESIS OF SCALE INHIBITORS FROM PINEAPPLE LEAF FIBER EXTRACT (ANANAS COMOSUS)
}

\author{
*Oyedeko K.F, Agbetan G.O, Akinyemi O.P
}

Department of Chemical Engineering, Lagos State University Epe Campus.

*kfkoyedeko@yahoo.com

Received: 20th July 2019

Accepted: $16^{\text {th }}$ December 2019

Published: $31^{\text {st }}$ March 2020

https://doi.org/10.47545/etrj.2020.5.1.058

\begin{abstract}
Scale inhibitors (SIs) are chemical substances that, when added to the scaling medium in little quantity mitigate or stops the further growth of scales. Scale is any crystalline deposit (salt) resulting from the precipitation of mineral compounds present in water. Preventing scale formation relies on understanding the composition of the scale, appropriate scale inhibitor, and early pretreatment. Many synthetic inhibitors are highly toxic to both humans and environment. Due to the hazardous effect of most of these inhibitors and restrictive environmental regulations, there have been need to focus on the development of natural renewable inhibitors that are environmentally friendly, ecologically acceptable, abundant and inexpensive. In this study Pineapple leaf is locally sourced and extracted to inhibit the growth of scale. The extract was prepared by dissolving $1 \mathrm{~g}$ of dry pineapple leaf powder in water with total volume of $1 \mathrm{~L}$ at a temperature of $90^{\circ} \mathrm{C}$. This solution was diluted, followed by crystallization process at operating condition of $85^{\circ} \mathrm{C}$. This process was followed by filtration and the residues obtained were weighed and recorded. The percentage inhibition rate of the plant material obtained gives an approximate value of $42.25 \%$. The study of utilization of Pineapple leave fiber (PALF) can be a new source of raw material to the industries and can be potential replacement of the expensive and nonrenewable synthetic chemicals. The advantage of natural fibers is their continuous supply, easy and safe handling, and biodegradable nature which are a characteristic to all green materials.
\end{abstract}

Keywords: Synthesis, Pineapple leaf, Scale inhibitor, Produced water

\section{INTRODUCTION}

The oil producing industry can be broken down into three key sections: Upstream, midstream and downstream. The upstream component is also known as the exploration and production (E and $\mathrm{P}$ ).

Midstream entails the transportation, storage and processing of oil and gas. Downstream refers to the refining and purifying of the raw materials obtained during the upstream phase. The marketing and commercial distribution of these products to end users [1].The varying amount of gases and the high concentration of salts in water are the major constituents of the oil and gas production industry. Many of these processes can eventually cause a problem. Whenever an oil or gas well produces water, or water is injected in the case of enhance recovery method, there is the possibility that scale will form.

Most common industrial processes, including petroleum production, mining, cooling water, desalination, reverse osmosis, pulp and paper manufacture, geothermal power production and sugar refining, operate using water systems and scale will only occur if water is produced [19]. This is because water is a solvent for the solubility to occur. For various reasons, scale forms during operations, such as mixing, heating, concentrating and evaporating water. Scale accumulation can cause huge losses in production, increasing costs and making the systems inefficient. Scale is any crystalline deposit (salt) resulting from the precipitation of mineral compounds present in water [18]. One of the important factors taken into account in the design of a crude oil treating system is the Scaling tendencies of the produced water since scale will occur only if water is produced. This is considered a problem as the formation of scale poses several serious menaces in oil and gas explorations [11]. The produced water from the crude oil well, which is naturally formed in the well or introduced into it during the oil recovery process. It can either exist separately from the crude oil or mix together with it. Scaling which occurs as a result of pressure, temperature, or composition changes 
in the solubility of the mineral constituents in the produced water causes a serious menace in the maintenance of equipment and production facilities. Work on the mechanism of scale formation by water in oil fields was done [12], [13] and suggests an accurate model capable of predicting scaling phenomena. The effect of magnetic water treatment on the scaling power of hard waters and shows how permanent magnets help to remove existing scale deposits in water [6]. Worked on Scale formation and control in oil and gas fields was carried out [10]. Inhibition techniques can range from basic dilution methods, to the most advanced and cost-effective methods of threshold scale inhibitors.[17].. The use of substances (scale inhibitors) that interact with the scales and prevent further growth of the scales is considered the simplest, practical and cost-effective technique. Scale inhibitors (SIs) are chemical substances that, when added to the scaling medium in little quantity mitigate or stops the further growth of scales. They are specialty chemicals that are added to oil production systems to delay, reduce and/or prevent scale deposition [2], [7].They can also be referred to as antiscalants. It is the ability of an antiscalant to keep supersaturated solutions of sparingly soluble salts. It prevents the precipitation of salts once the salt has exceeded its solubility product.

The natural product extracts are viewed as an incredibly rich source of naturally synthesized chemical compounds that can be extracted by simple procedures with low cost, and are biodegradable in nature. This area of research is of much importance because in addition to being environmentally friendly and ecologically acceptable, plant products are inexpensive, readily available and renewable source of materials. This gives a push for 'green' products to be employed in the preparation of scale inhibitors. Providentially, some of the active functionalities present in these compounds could be obtained from isolates or phytochemicals in extracts of plant materials. Research on the use of plant materials as oilfield chemicals like drilling mud [8] biosurfactants [15] and corrosion inhibitors [9] is very active, but very little has been done to test some plant materials as SIs. Extracts of natural plants materials are environmentally friendly, non-toxic, relatively less expensive, readily and sustainably available and also biodegradable. Nevertheless, this biodegradability limits the storage and long-term usage of plant extracts/local materials. The decomposition of plants extracts by microorganisms can be prevented by addition of biocides and other stabilizing agents. Therefore, some plant extracts could be blended with biocides to obtain effective scale inhibitors. In an ideal system, a very small amount of chemical injected, would amount to the total disappearance of the scale Scale inhibitors which include plant materials and biodegradable polymer and insulin derivatives exhibit a large inhibiting properties trace. Various natural products, especially from plants, contain generous amounts of polyphosphates, carboxylic acid groups, alcohol and aromatic amines, which are potential functionalities for adsorption or antiscaling effect of the chemical SIs. Plants materials reported as efficient scale inhibitors include sea weeds polysaccharides and soybean oil methyl ester olive leaf extract and leaf extracts of Punicagranutum. There are also reports on products or compounds from plants and natural products such as humic acid, leucine, citric acid and xanthan used as scale inhibitors [4], [5]. Some potential plant materials have also been suggested but yet to be tapped into for research and experimentation. For this research, the category of plant materials chosen can be classified in the same realm as wood. Various researchers, has also carried out different experiment in this regard. They have synthesis products from green extract to actualize this and also, they have been able to show how efficient these inhibitors are in terms of percentage. Piper betle leaf extract as a green inhibitor of calcium sulphate $\left(\mathrm{CaSO}_{4}\right)$ scale formation. The ability of Piper betle leaf extract as an inhibitor of the formation of $\mathrm{CaSO}_{4}$ is $47.07 \%$. [14]. In this study scale inhibitors from green extract (pineapple leaf) was focused on effectively achieving the, formulation of inhibitor from green extracts (pineapple leaf) whose analysis has been shown to have scale deposit problem or has been subjected to such condition by varying some of its physical properties such as; Temperature (T) and Pressure (P). It is of great desire to look at this area as much research has not been made towards this end. Although, other materials had been used but not the commonly sourced ones. This study is done, so as to maximize the full utilization of the abundant green materials present in nature and to reduce the pressure on other available resources.

The pineapple is a stoloniferous herbaceous, short-lived perennial plant that can reach $0.75-1.25 \mathrm{~m}$ in height, with a short and thick stem and a very shallow root. The leaves are waxy, succulent, $50-180 \mathrm{~cm}$ long and sword-like, and bear sharp upcurved spines on the edges. Pineapple leaf extract is a source of natural fibers. Natural fiber-based composites are ecofriendly in nature and have some peculiar properties attributed to them. Pineapple leave fiber (PALF) is one of the abundantly available wastes materials of Malaysia and has not been studied yet as it is required [3],[16] also in Nigeria. 


\section{MATERIALS AND METHOD}

\subsection{Materials}

The following materials were used; $560 \mathrm{ml}$ produced water (obtained from DPR), pineapple leaves (locally sourced), deionized water. $250 \mathrm{ml}$ Nalgene polypropylene bottles (12), sieve, blender.

The apparatus used are; measuring cylinder, weighing balance, water bath, desiccator, filter paper, funnel, heating oven, heating mantle, beakers. Also, for the Data Analysis part of this work MS EXCEL 2016 was used in the plotting of graphs.

\subsection{Experimental Procedure}

Analysis of the produced water: Laboratory analysis was carried out on the produced water to determine the mineral cations present in it. This analysis was carried out at Lagos State Environmental and Protection Agency (LASEPA) at Ikeja. This analysis is aimed to analyze and quantify in terms of concentration the cations available in the sample of the produced water while the LASEPA standard was used as a basis.

This analysis was a confirmatory test to the presence of calcium residue in the water, these are main factors to scale deposition

Preparation of pineapple leaf extract: Phytochemical analysis was done by taken the Pineapple leaf composition that was sourced from LASU Epe campus and was taken to laboratory for analysis in other to characterize the properties peculiar to them and to rate their \% composition. As the plant source, species, and geography are great factors that also contribute to the qualities of chemical properties of the pineapple leaf.

Preparation of pineapple leaf was made by dissolving of $1 \mathrm{~g}$ dry Pineapple leaf powder in water with total volume of $1 \mathrm{~L}$ at temperature of $90^{\circ} \mathrm{C}$ for $1 \mathrm{hr}$ and it was kept for 1 night. Furthermore, 1000ppm of Pineapple leaf extract solution obtained was diluted to 450ppm.

Extraction process in details: The pineapple leaves were sourced and obtained within the campus, (LASU EPE), they were sorted out to separate the fresh from the dried into a separate container, the leaves were collected and rinse so as to remove all dirty particles hanging to the body. The cleaned, fresh leaves were then put into the oven for drying the operating condition was set to $225^{\circ} \mathrm{C}$ for up to $5 \mathrm{hrs}$ so as to ensure uniformity. The oven was autoclave at $120^{\circ} \mathrm{C}$ for 15mins. to kill all existing microorganisms that might possible interfere with the plant leaves to be dried. The dried leaves were then collected and crushed and blended into powder form. The crushed leaves were then further sieved to remove the big particles out of it; hence, smoother fine particle was further collected. One liters (1L) of water was heated on a heating mantle for operating condition of $90^{\circ} \mathrm{C}$ for $1 \mathrm{hr}$ The temperature of this water was monitored using a thermometer until the $90^{\circ} \mathrm{C}$ temperature is established and maintained. The measured $1 \mathrm{~g}$ powder of the pineapple leave was then added to the water, continuous stirring is done to achieve uniformity in the solution. After the completion of one hour from step (iii) above, some of the physical properties of this solution changes; in terms of color, odor and some other peculiar properties. The solution was left for one night so as to make the concentration more toxic and well aligned. The obtained solution was filtered and clean, bright urine like solution was then obtained. The prepared green inhibitor was put in the wash bottle for easy application and precision and was separately added into each bottle to have in all 12 prepared solutions with added inhibitors. The effect of adding additive was determined by adding additive of $450 \mathrm{ppm}$ into the prepared solution which has been detected to contain $\left(\mathrm{Ca}^{2}+\right)$. The weight of the crystals was measured and the amount precipitated was calculated.

The volume of the produced water was measured in $45 \mathrm{ml}$ into 12 different $250 \mathrm{ml}$ of polypropylene bottles. This type of bottle is selected for this process because, they have the ability to withstand the temperature condition designed for this part of the process. Six out these bottles are to serve the purpose of control; meaning no additives was added to 
them. To the remaining six bottles containing $45 \mathrm{ml}$ of the produced water, the additives (prepared solution of the inhibitor) was added (at least 4 times press of the wash bottle was poured). Crystallization experiment was carried out by putting the obtained water into $250 \mathrm{ml}$ polypropylene bottles, each containing $45 \mathrm{ml}$ ( 6 bottles) in two different portions. The bottles were then put into the water bath at a temperature of $85^{\circ} \mathrm{C}$. Over the 72 min experiment, a bottle was taken every $12 \mathrm{~min}$. the precipitate was washed thoroughly with deionized water and dried at a temperature of $102^{\circ} \mathrm{C}$ in the oven until dry. The weight of the crystals was measured and the amount precipitated was calculated. With each crystallization observation, a blank containing no additives was carried out in conjunction with the additive experiments.

Data analysis: were obtained as the amount of precipitate versus time at $0.05 \mathrm{M}$ concentration of the solution and of $470 \mathrm{ppm}$ additive added, and it was plotted as the amount of precipitate versus time using MS Excel 2016. The effectiveness of the additive in inhibiting the desired (crystal) scale formation can be determined from the mass of precipitate obtained and the results were fully discussed and detailed.

\section{RESULTS AND DISCUSSION}

\subsection{Analysis of Results Obtained}

The Table 1 shows the break down in w/w\% the general constituents of a pineapple leaf and this composition are the primary considerations that characterize the leave as a function of scale inhibitors.

Table 1: Constituents /Compositions of a pineapple leaf

\begin{tabular}{ll}
\hline Constituents/ Composition (w/w \%) & Pineapple Leaf \\
\hline Ash Contents & $4.50^{*}$ \\
Cellulose Contents & $66.2 *$ \\
Holocellulose & $85.7 *$ \\
Hemicellulose & 19.5 \\
$1 \% \mathrm{NaOH}$ solubility & $39.8^{*}$ \\
Lignin Contents & $4.28^{*}$ \\
Moisture Contents & 81.6 \\
(Note: $*$ favourable value) & \\
\hline
\end{tabular}

The results obtained from the experiment explains the effect of the inhibitor added with trend in concentration and time. The results were further use in calculating the efficiency of the inhibitor prepared.

During the crystallization process, the precipitate formed as a result of changes in time under a constant temperature $\left(85^{\circ} \mathrm{C}\right)$ was recorded and tabulated and a graph was plotted on excel(Table3, and 4$)$. The analysis on the produced water at LASEPA was done and the result generated quantifies in concentration the amount of the different cations presents in the water samples as obtained in Table2. Also, to generate Table 3 same process was followed only that in this case 470 PPM solution of the prepared inhibitor was added. The results (i.e. precipitate was obtained as a function of time) was tabulated and plotted using excel so as to further explain the trend.

Table 2: Result analysis on Produced Water

\begin{tabular}{llccl}
\hline S/N & $\begin{array}{l}\text { TRACE/TOXIC } \\
\text { HEAVY METAL }\end{array}$ & $\begin{array}{l}\text { RESULT } \\
(\mathbf{m g} / \mathbf{l})\end{array}$ & $\begin{array}{l}\text { STANDARD } \\
(\mathbf{m g} / \mathbf{l})\end{array}$ & REMARKS \\
\hline 1 & Calcium & 226.4519 & 200 & High \\
2 & Zinc & 0 & 2 & \\
3 & Iron & 2566.911 & 10 & Very high
\end{tabular}



$4 \quad$ Sodium
18.9523
5 Manganese
0.1805
5

Table3: Values of Weighed Precipitates against Time at (0 PPM) Additives

\begin{tabular}{cccc}
\hline SAMPLES & $\begin{array}{c}\text { TEMPERATURE } \\
\left({ }^{\circ} \mathbf{C}\right)\end{array}$ & $\begin{array}{c}\text { TIME } \\
(\text { MIN })\end{array}$ & $\begin{array}{c}\text { PRECIPITATION } \\
\text { MASS CHANGE } \\
(\mathbf{g} / \mathbf{l})\end{array}$ \\
\hline A & 85 & 12 & 0.98 \\
B & 85 & 24 & 1 \\
C & 85 & 36 & 1.04 \\
D & 85 & 48 & 1.01 \\
E & 85 & 60 & 1.01 \\
F & 85 & 72 & 1.03 \\
\hline
\end{tabular}

Table4: Values of Weighed Precipitates against Time at (470 PPM) Additives

\begin{tabular}{ccrc}
\hline SAMPLES & $\begin{array}{l}\text { TEMPERATURE } \\
\left({ }^{\circ} \mathrm{C}\right)\end{array}$ & $\begin{array}{l}\text { TIME } \\
(\mathrm{MIN})\end{array}$ & $\begin{array}{l}\text { PRECIPITATION } \\
\text { MASS CHANGE } \\
(\mathrm{g} / \mathrm{l})\end{array}$ \\
\hline $\mathrm{A}$ & 85 & 12 & 0.99 \\
$\mathrm{~B}$ & 85 & 24 & 1.01 \\
$\mathrm{C}$ & 85 & 36 & 1.03 \\
$\mathrm{D}$ & 85 & 48 & 1.01 \\
$\mathrm{E}$ & 85 & 60 & 1.01 \\
$\mathrm{~F}$ & 85 & 72 & 1 \\
\hline
\end{tabular}

\subsection{Graphical Presentation of the results obtained.}

Excel was used as the software tool for plotting of graphs in this work. The results were plotted on graphs to show the trend whether linear, cyclic or otherwise in the inhibition process of the growth crystals. 


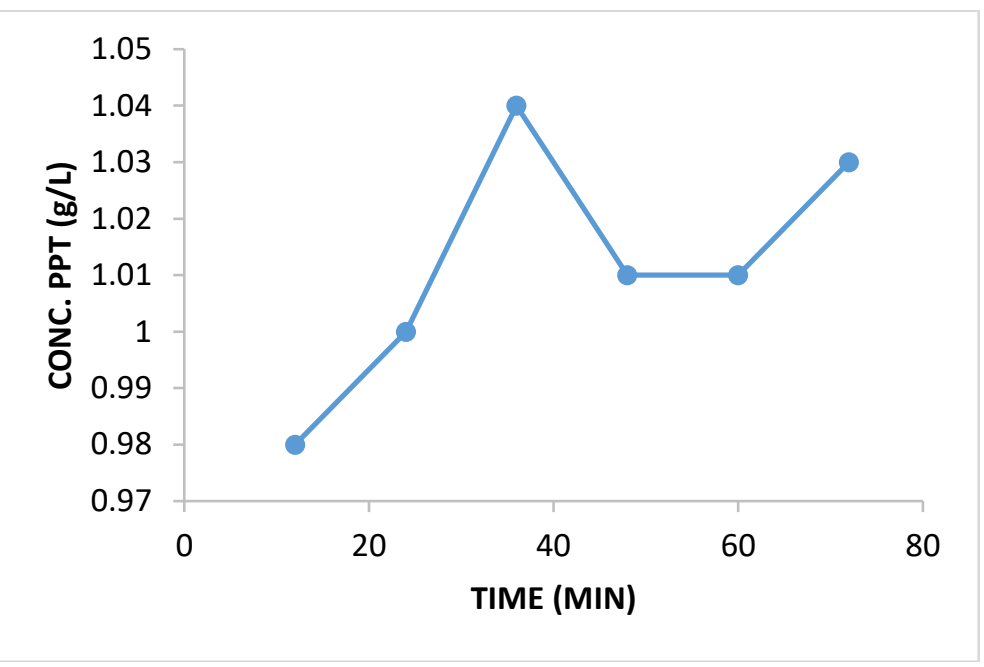

Figure 1: Graph of Concentration against Time at $0 \mathrm{ppm}$ of additives.

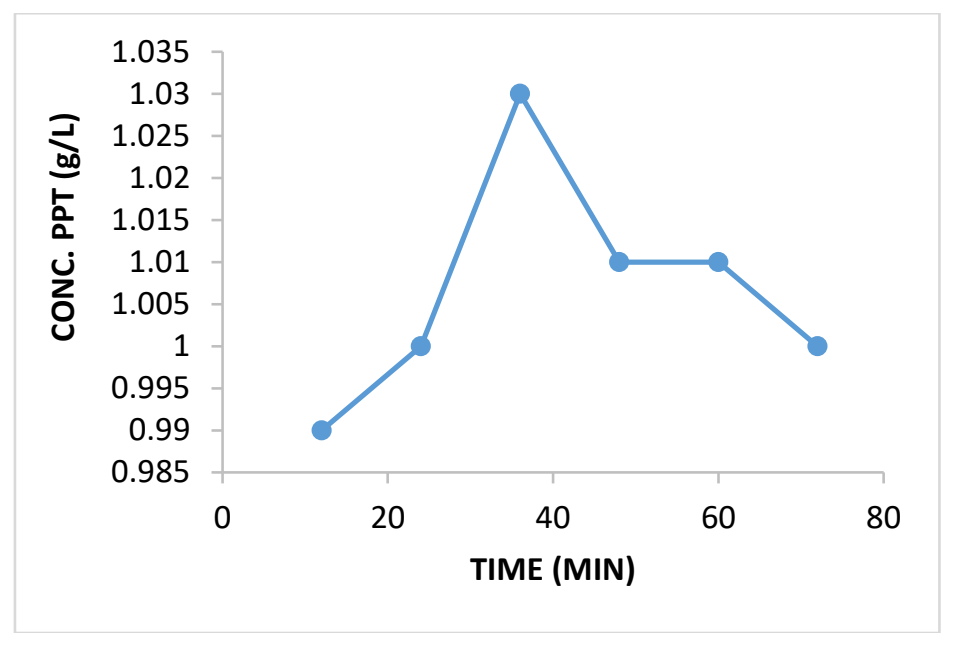

Figure 2: Graph of Concentration against Time at 470ppm of additives.

Inhibitor Efficiency Calculation: Percentage of Inhibitor Efficiency (IE \%) is a measure of the ability of an inhibitor to inhibit crystal growth. The greater the IE\% value, the better the inhibitory power. Based on these equations, inhibitor efficiency of Pineapple leaf extract at a concentration of $470 \mathrm{ppm}$ and the concentration of $1.101 \mathrm{~g} / \mathrm{L}$ growth solution with an inhibition time of 0-72 minutes as shown in Table 5 and Fig. 3.

The efficiency of the inhibitor prepared was calculated using the values in Tables 3 and 4 . The percentage efficiency at different time and at different weight in concentration of the precipitates.

To calculate the efficiency, we use the expression;

$$
\% I E=\frac{C a-C b}{C o-C b} \times 100
$$

Where; $\mathrm{C}_{\mathrm{a}}=$ Precipitation amount of calcium compound after added inhibitor at equilibrium $(\mathrm{g} / \mathrm{L})$ $\mathrm{C}_{\mathrm{b}}=$ Precipitation amount of calcium compound without inhibitor at equilibrium $(\mathrm{g} / \mathrm{L})$ 
$\mathrm{C}_{\mathrm{o}}=$ Initial Precipitation amount of Calcium Compound $(\mathrm{g} / \mathrm{L})=1.101 \mathrm{~g} / \mathrm{L}$

Table 5: Recorded values of the inhibitor percentage with change in time.

\begin{tabular}{ccc}
\hline $\mathbf{C a}$ & $\mathbf{C b}$ & \% INHIBITION \\
\hline 0.99 & 0.98 & 8.333 \\
1 & 1 & 0 \\
1.03 & 1.04 & 16.667 \\
1.01 & 1.01 & 0 \\
1.01 & 1.01 & 0 \\
1 & 1.03 & 42.86 \\
\hline
\end{tabular}

From the experiment it was observed that the amount of $\mathrm{Ca}^{2+}$ compound precipitate increases with the increasing of contact time until it reaches equilibrium. The inhibition of $\mathrm{Ca}$ compound growth by pineapple leaf fiber extract can be seen from the reduced amount of $\mathrm{Ca}^{2+}$ compound which settles in the solution added to the inhibitor. The optimum inhibition of $\mathrm{Ca}^{2+}$ by pineapple leaf fiber extract occurred at 72 minutes of contact time.

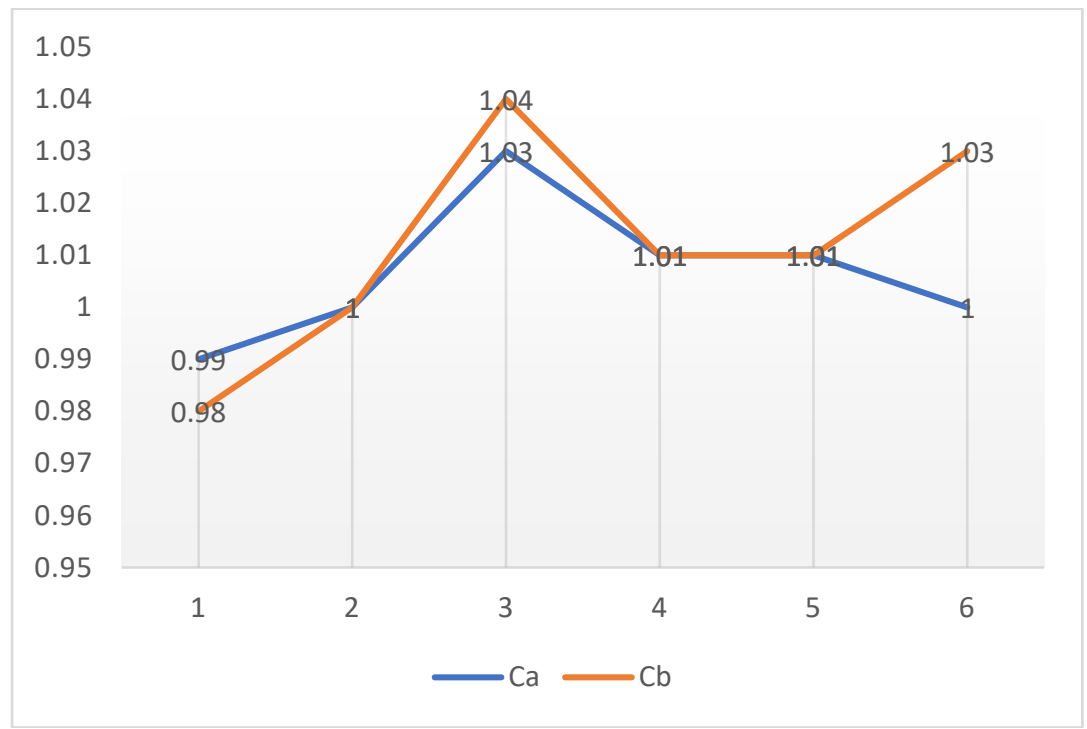

Figure 3: Graph of Concentration at 0ppm to 470ppm additives.

The samples indicated to have OPPM additives in it were taken as control for the process. The trend clearly showed that there was an increase in the amount of precipitate formed as the operation progresses with time at that operating condition of $85^{\circ} \mathrm{C}$; this was noted at the first $36 \mathrm{mins}$ the process began. At a particular time, the amount of precipitate deposited was constant, (i.e. equilibrium sets in). As the process proceeds out of this phase, the precipitates formed keep increasing as time progresses upward. From results onTable4, and shown in fig 2 after introducing 470PPM sample of the inhibitor into the produced water; at the earlier time into the experiment, there was no noticeable change in the amount of precipitate deposited as that from the control at same time range. As the process progresses with time, there is a decrease in the amount of precipitate formed when compared to the control process at same time range. At a point, the concentration of the precipitates formed was constant at same point similar to that from the control process, coming out of this phase; the amount of precipitates formed reduces drastically which duly satisfy the aim of this experimental project. 
The efficiency of the inhibitor calculated is very low and impractical when compared to that obtained in previous literature review revised; reason being that the initial amount of calcium ion $\left(\mathrm{Ca}^{2+}\right)$ in the produced water is of very small quantity. The maximum efficiency of the inhibitor was calculated using equation 1 as $42.253 \%$ this is in agreements with most authors [3],[5],[14 and [19].

\section{CONCLUSION}

This study gives solutions to the steps on how to make or produce the scale inhibitor from Pineapple leaf fiber and the effectiveness of the green scale inhibitor. These resources extracted from nature (plant materials/green extract) was used to inhibit the formation of scale which tends to be one of the major problems faced in the Oil and gas industry.

The progressive trend in time has been seen as a major factor that contributes to the deposition of residual scale components in water. Hence, the best time were the effective action of the inhibitor was recorded should be adequately considered as an option while introducing the inhibitor into the water.

The results obtained have shown that Pineapple leaf extract can act as an inhibitor of Ca compound deposit formation. This process was carried out using the adsorption mechanism. The inhibitor effectiveness of Pineapple leaf extract at a concentration of $470 \mathrm{ppm}$ and the concentration of growth solution of $1.101 \mathrm{~g} / \mathrm{L}$ is $42.253 \%$. This work is cost effective and environmentally suitable for practice on medium scale practice and can as well be pilot into industrial scale process. It is recommended that a detailed study of chemical, physical, and mechanical properties will bring out logical and reasonable utilization of pineapple leaf fiber for various applications. From environmental and economic perspective. Also, it can be a new source of raw material to the industries. Considering local content view it can be a potential replacement of the expensive and nonrenewable synthetic chemicals.

\section{REFERENCES}

[1] Alfred D. Chandler, Jr. Shaping the Industrial Century - The Remarkable Story of the Evolution of the Modern Chemical and Pharmaceutical Industries. (Havard University Press, 2005).

[2] Alzahrani, Salem; Mohammad, Abdul Wahab (2014). "Challenges and trends in ba membrane technology implementation for produced water treatment: Journal of water process Engineering. 4: 107-133.

[3] Asim. M, Khalina Abdan, . Jawaid. M, Nasir. M, Zahra Dashtizadeh, Ishak, M.R and Enamul Hoque A M (2001) Review on Pineapple Leaves Fibre and Its Composites, Natural Fiber Reinforced Polymer Composites

[4] Chauhan K, Sharma P and Chauhan G S (2015) Mineral Scales and Deposites 701-720 Côté, W.A. (1968). Chemical composition of wood. In Principles of Wood Science and Technology, Springer Berlin Heidelberg. 55-78

[5] Chaussemier, M ,Ermane Pourmohtasham, Dominique Gelus, Nathalie Pecoul, Hubert Perrot, Jean Lédion, Hélène Cheap-Charpentier, ,Olivier Horner, (2015) State of art of natural inhibitors of calcium carbonate scaling. A review article Research gate publication

[6] El Hafidi,M.Y. M. El Hafidi (2017) Existing Scale Deposits Removal by Magnetic Water Treatment: Theoretical Study and Experiment. Int J Water Wastewater Treat 3(3): http://dx.doi.org/10.16966/2381-5299.143

[7] Frenier,W., Wayne (2008). Formation, removal, and inhibition of inorganic scale in the oilfield

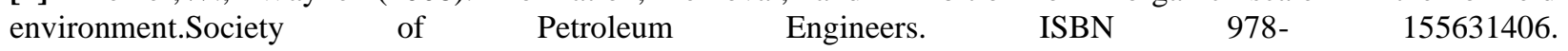
http://catdir.loc.gov/catdir/toc/fy12pdf01/2009517707.html: 
[8] Ghazali. Wan Nor MaawaWan , RizalmanMamat, H.H.Masjuki Gholamhassan Najafi (2015) Effects of biodiesel from different feedstocks on engine performance and emissions: A review Renewable and Sustainable Energy Reviews Volume 51, November 2015, Pages 585-602

[9] Ituen, E.B, Akaranta,O, Umoren.S,A , (2017). Eco-friendly corrosion inhibitor from Pennisetum purpureum ...Journal of Molecular Liquids 246, 112-118, 2017. 37

[10] Jianbo Li, Mingjin Tang, Zhengrong Ye, Longli Chen and Yuqin Zhou, (2017). Scale formation and control in oil and gas fields, A review, Journal of Dispersion Science and Technology, 38:5, 661-670, DOI: $\underline{10.1080 / 01932691.2016 .1185953}$

[11] Jian Hao, Lingyun Li, Weiwei Zhao, Xiaqian Wu, Yangyang Xiao, Hongfeng Zhang, Na Tang, Xiaocong Wang. Synthesis and Application of CCQDs as a Novel Type of Environmentally Friendly Scale Inhibitor. ACS Applied Materials \& Interfaces2019,11 (9), 9277-9282. DOI: 10.1021/acsami.8b19015.

[12] Moghadasi, J., M. Jamialahmadi, H. Müller-Steinhagen and A. Sharif. 2003a. Scale formation in oil reservoir and production equipment during waterinjection (Kinetics of $\mathrm{CaSO} 4$ and $\mathrm{CaCO} 3$ crystal growth and effect on formation damage). In: SPE European Formation Damage Conference: Society of Petroleum Engineers.

[13] Moghadasi, J., M. Jamialahmadi, H. Müller-Steinhagen, A. Sharif, A. Ghalambor, M. Izadpanah and E. Motaie. 2003b. Scale formation in Iranian oil reservoir and production equipment during water injection. In: International Symposium on Oilfield Scale: Society of Petroleum Engineers.

[14] Santoso P. , Setiawan M.R., Suharso (2019)Piper betle leaf extract as a green inhibitor of calcium sulphate (CaSO4) scale formation IOP Conf. Series: Earth and Environmental Science 258 (2019) 012038

[15] Silva, R. D. C. F., Almeida, D. G., Rufino, R. D., Luna, J. M., Santos, V. A., Sarubbo, L. (2014). Applications of biosurfactants in the petroleum industry and the remediation of oil spills. International journal of molecular sciences, 15(7), 12523-12542.

[16] Taj,S., M. A. Munawar, and S.U Khan, "Natural fiber-reinforcced polymer composites," Proceedings of the Pakistan Academy of Sciences, 44: 120-133, 2006.

[17] Tomson MB, Fu G, Watson MA, Kan AT (2002) Mechanism of mineral scale inhibition, society of petroleum engineers. Oilfield Scale Symposium, Aberdeen.

[18] Tung A. Haong. Mechanisms of scale Formation and Inhibition. 2015, 47-83. Do1: 10.1016/B978-9.00003-6.

[19] Zhang, Z; Stout, J.E., Vidic, R.D., 2006. The impact of temperature and TOC on chlorine dioxide decay in drinking water. American water works Association Annual,San Antonio, TX. 